\title{
Identification of differentially expressed genes involved in early bolting of Angelica sinensis (Apiaceae)
}

\author{
G. Yu' ${ }^{1}$ Y.X. $\mathrm{Ma}^{2}$, J.-A. Duan' ${ }^{1}$ B.S. Song ${ }^{3}$ and Z.Q. $\mathrm{He}^{3}$ \\ ${ }^{1}$ Nanjing University of Chinese Medicine, Nanjing, China \\ ${ }^{2}$ China Pharmaceutical University, Nanjing, China \\ ${ }^{3}$ Gansu Mingui Chinese Medicinal Materials Technology Co., Ltd., \\ Lanzhou, China \\ Corresponding author: J.-A. Duan \\ E-mail: dja@njutcm.edu.cn
}

Genet. Mol. Res. 11 (1): 494-502 (2012)

Received August 2, 2011

Accepted January 23, 2012

Published March 6, 2012

DOI http://dx.doi.org/10.4238/2012.March.6.2

\begin{abstract}
Angelica sinensis is a highly valued medicinal herb, known as female ginseng that is widely cultivated in China. Although A. sinensis is in great demand due to its multiple medicinal and food applications, its early bolting rate (almost $40 \%$ ) seriously affects crop quality. To better understand its flowering mechanism, cDNA-amplified RFLP analysis was employed to look for gene expression differences between flower bud and shoot apical meristem tissues. Sixty-four primer sets were used, with each primer set amplified to 60 transcriptderived fragments. Some transcript-derived fragments were expressed only in the flower bud. After cloning, sequencing and a homology search, 46 distinct sequences were obtained; 26 of these were found to have homologous sequences in databases. These included transcaffeoyl-CoA 3-O-methyltransferase, 1-deoxy-D-xylulose 5-phosphate reductoisomerase, 15-cis-zeta-carotene isomerase, isoamylase, and calmodulin-binding protein. These genes are closely related to pollen germination and pollen tube growth, terpenoid backbone biosynthesis, and other metabolic pathways. Confirmation of differential expression
\end{abstract}


of 10 sequences was obtained by semi-quantitative RT-PCR, showing higher expression levels in flower buds.

Key words: cDNA-AFLP; Early bolting; Angelica sinensis Diels; TDFs; sqRT-PCR; Female ginseng

\section{INTRODUCTION}

Angelica sinensis (Oliv.) Diels (Umbelliferae) is a world-renowned medicinal herb found in China (mainly in Gansu Province), Japan, and Korea. The radix of A. sinensis, called Danggui, is a popular tonic recorded as early as AD 25-225 in the book Shen Nong Ben Cao Jing during the Han Dynasty. It is recommended for the treatment of amenorrhea and dysmenorrhea, as an antiinflammatory agent in menstrual disorders, and as a hematopoietic and tonic, among other uses (Nanjing University of Chinese Medicine, 2006; Wang et al., 2007). It is also a dietary supplement in Europe and America and a health food product for women in Asia (Yang et al., 2008). Because of this wide variety of food and medicinal applications, demand for A. sinensis is increasing.

In Gansu Province where the herb originated, however, its quality has been seriously affected by its high early bolting rate, restricting its further development. Flowering affects the accumulation of medicinal compounds in A. sinensis, reducing its effectiveness as materia medica. Scientists have, therefore, begun to focus on the ecological factors involved in early bolting as well as the nutritional status of the plant itself (Lin and Qiu, 2007; Wang et al., 2007) rather than on specific molecular mechanisms. Currently, no effective methods for the control and prevention of this characteristic exist, although the intense demand for the plant presses for an urgent solution.

Through studies of Arabidopsis, rice, and other model plants, researchers have found that bolting is affected by gene expression and environmental factors. Four pathways affect flowering time: the photoperiod, vernalization, autonomous, and gibberellin pathways (Yanovsky and Kay, 2003; Komeda, 2004). These pathways have not been yet studied in A. sinensis. Complementary DNA-amplified fragment length polymorphism (cDNA-AFLP) analysis with high reproducibility can be used to screen a large number of differentially expressed cDNAs systematically (Money et al., 1996; Habu et al., 1997; Milioni et al., 2002). In the present study, cDNA-AFLP analysis was carried out to identify the differentially expressed genes in early bolting and normal A. sinensis. Several A number of candidate genes that might be involved in early bolting were identified.

\section{MATERIAL AND METHODS}

\section{Plant materials}

A. sinensis, a triennial medicinal plant, is characterized by early bolting, which frequently occurs in May. Thus, since 2004, and each year after this period, flower buds and sprout-shoot apical meristems were gathered from plants in Gansu Province.

\section{RNA preparation and cDNA synthesis}

Flower buds of early bolting plants, each approximately $0.5 \mathrm{~cm}$, were harvested before full blooming together with sprout-shoot apical meristems from normal plants. The samples 
were frozen in liquid nitrogen and stored at $-80^{\circ} \mathrm{C}$. Total RNA was extracted from the plant materials using the Trizol extraction method (Invitrogen, USA). RNA integrity and quantity were determined by running $2 \mu \mathrm{L}$ total RNA in a formamide denaturing gel. First- and second-strand cDNA synthesis was obtained using a Universal Riboclone cDNA Synthesis System (Promega, USA), according to manufacturer instructions. The resulting double-stranded cDNA was separated on agarose gel to check the sizes of the cDNA samples.

\section{Preparation of the samples for cDNA-AFLP analysis}

cDNA-AFLP was carried out according to the procedure of Habu et al. (1997) with minor modifications. Approximately $500 \mathrm{ng}$ double-stranded cDNA was subjected to standard AFLP template production. The restriction enzymes used for the digestion of cDNA were MseI (10 U/ $\mu \mathrm{L}$; Sangon, China) and EcoRI (10 U/ $\mu \mathrm{L}$; Sangon). The digested products were ligated to adapters with the following sequences: MseI adapter, 5'-GACGATGAGTCCTGAG-3' and 5'-TACTC AGGACTCAT-3'; EcoRI adapter, 5'-CTCGTAGACTGCGTACC-3' and 5'-AATTGGTACG CAGTC-3'. The ligated products were preamplified with the corresponding preamplification primers (MseI: 5'-GATGAGTCCTGAGTAA-3'; EcoRI: 5'-GACTGCGTACCAATTC-3'). Twenty cycles of preamplification were performed in a MyCycler PCR system (Bio-Rad, USA) as follows: $30 \mathrm{~s}$ at $94^{\circ} \mathrm{C}, 30 \mathrm{~s}$ at $56^{\circ} \mathrm{C}$, and $1 \mathrm{~min}$ at $72^{\circ} \mathrm{C}$. The final template was diluted 50 -fold with sterile water in preparation for selective amplification.

\section{cDNA-AFLP reactions and polyacrylamide gel electrophoresis of the selectively amplified products}

Equal amounts of preamplified products were amplified using primers with selective nucleotides. The primers used for selective amplification were as follows: EcoRI+NN primer, 5'-GACTGCGTACCAATTCNN-3' and $M s e I+N N$ primer, 5'-GATGAGTCCTGAGTAANN-3', in which $\mathrm{N}$ could correspond to A, C, G, or T. The first cycle consisted of $30 \mathrm{~s}$ at $94^{\circ} \mathrm{C}, 30 \mathrm{~s}$ at $65^{\circ} \mathrm{C}$, and $1 \mathrm{~min}$ at $72^{\circ} \mathrm{C}$. For the next 13 cycles, the annealing temperature was reduced by $0.7^{\circ} \mathrm{C}$ per cycle. The final 23 cycles consisted of $30 \mathrm{~s}$ at $94^{\circ} \mathrm{C}, 30 \mathrm{~s}$ at $56^{\circ} \mathrm{C}$, and $1 \mathrm{~min}$ at $72^{\circ} \mathrm{C}$. Four sets of the EcoRI primer and 16 sets of the MseI primer were combined to give a total of 64 primer pair combinations. For high-throughput analysis of differentially expressed transcriptderived fragments (TDFs), the polymerase chain reaction (PCR) products were separated on denaturing $6 \%$ polyacrylamide gels. The differentially amplified bands were cut directly from the gels on the basis of the scanned image.

\section{TDF isolation and reamplification}

The polymorphic TDFs identified based on presence and expressed at higher levels in the flower buds were cut from the denaturing $6 \%$ polyacrylamide gel. They were initially eluted in $50 \mu \mathrm{L}$ sterile double-distilled water at $95^{\circ} \mathrm{C}$ for $15 \mathrm{~min}$, and then hydrated overnight at $4^{\circ} \mathrm{C}$.

Two microliters of the aliquot was used for reamplification to a total volume of $20 \mu \mathrm{L}$ with the same set of corresponding selective primers and PCR conditions as those used for selective amplification except that an annealing temperature of $56^{\circ} \mathrm{C}, 35$ cycles, and a final 5 -min extension were used. The PCR products were resolved on a 1.5\% $1 \mathrm{X}$ Tris-acetate ethylenediaminetetraacetic acid-agarose gel, and each single band was isolated and eluted using a Takara gel extraction kit (Takara, Japan). The eluted TDFs were cloned into a plasmid PMD- 
$18 \mathrm{~T}$ vector (Sangon) following the manufacturer protocol.

\section{Sequence analysis}

Five colonies were selected for the sequencing analysis of each TDF. Sequencing was carried out using an automated sequencer. The sequences of the TDFs were analyzed for their homology against nonredundant genes, expressed sequence tags (ESTs), or transcripts in public databases (http://www.ncbi.nlm.nih.gov/BLAST, http://www.arabidopsis.org/cgibin/fasta/nph-TAIRfasta.pl, and http://www.shigen.nig.ac.jp/rice/oryzabase/blast) using the BLASTN and BLASTX algorithms (Altschul et al., 1997). The homologous sequences were further analyzed in databases (http://www.genome.jp/kegg/ and http://www.geneontology. org/) to understand their function more fully.

\section{Semi-quantitative reverse transcription PCR (sqRT-PCR)}

sqRT-PCR was carried out on $2 \mu$ g total RNA using a two-step RT-PCR kit (Takara, Japan) according to manufacturer instructions. Primers were designed from the sequences of the TDFs using a Primer 3.0 web resource (http://frodo.wi.mit.edu/). Total RNA was used as the internal standard in the RT-PCR for checking the quality of the RNA template. PCR conditions were essentially the same as those described earlier for the reamplification of the TDFs. To obtain reproducibility of results, we repeated the sqRT-PCR analysis three times.

\section{RESULTS}

\section{cDNA-AFLP fingerprinting}

A total of 64 primer combinations (Table 1) were selected for cDNA-AFLP. On average, 60 clear and unambiguous bands (TDFs) were generated with each primer combination. Although most combinations of primers produced identical patterns, some TDFs were expressed at a higher level in the flower bud (Figure 1). This difference implied that minor changes in gene expression could account for the phenotypes of early bolting observed in $A$. sinensis. These differentially expressed TDFs were successfully recovered from the denaturing polyacrylamide gels for further analysis.

Table 1. Sequences of the primers used for amplified restriction fragment length polymorphism.

\begin{tabular}{ll}
\hline Mse I+NN & $E c o$ RI+NN \\
\hline GATGAGTCCTGAGTAAAA & GACTGCGTA CCAATTCCA \\
GATGAGTCCTGAGTAAAT & GACTGCGTA CCAATTCCT \\
GATGAGTCCTGAGTAAAC & GACTGCGTA CCAATTCCC \\
GATGAGTCCTGAGTAAAG & GACTGCGTA CCAATCCG \\
GATGAGTCCTGAGTAATA & \\
GATGAGTCCTGAGTAATC & \\
GATGAGTCCTGAGTAATG & \\
GATGAGTCCTGAGTAATT & \\
GATGAGTCCTGAGTAACA & \\
GATGAGTCCTGAGTAACT & \\
GATGAGTCCTGAGTAACC & \\
GATGAGTCCTGAGTAACG & \\
GATGAGTCCTGAGTAAGA & \\
GATGAGTCCTGAGTAAGT & \\
GATGAGTCCTGAGTAAGC & \\
GATGAGTCCTGAGTAAGG & \\
\hline
\end{tabular}




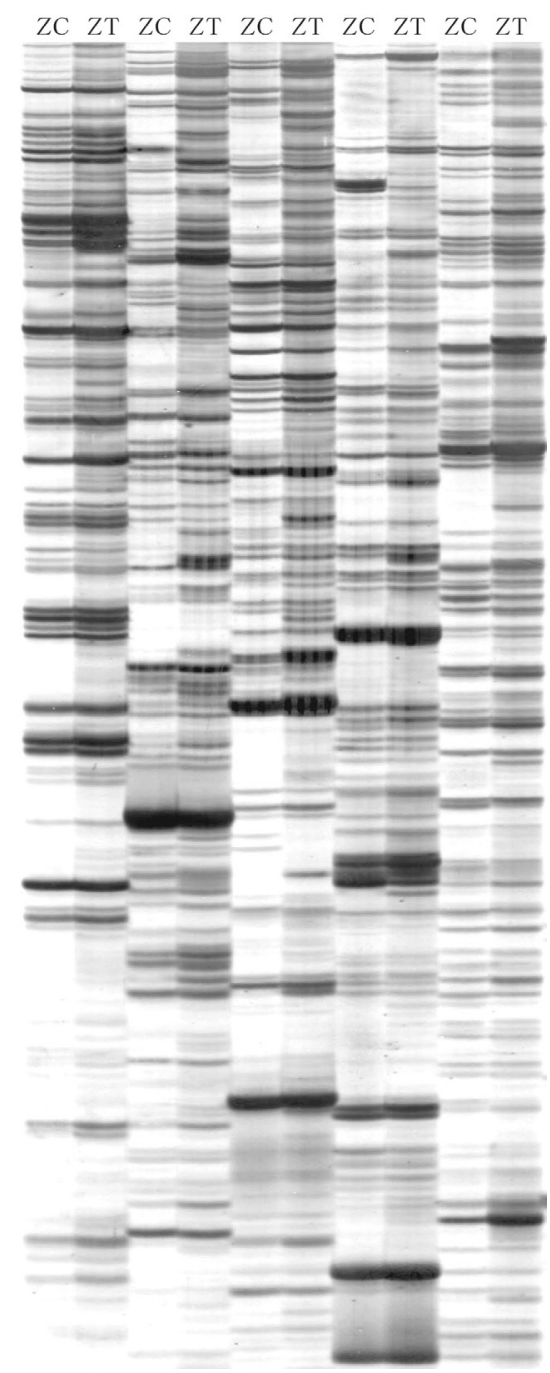

Figure 1. cDNA-AFLP autoradiogram of five of the most information primer combinations amplifying differentially expressed genes in flower bud and shoot apical meristem. The combinations of primers used were included according to the codes reported in Table $1 . \mathrm{ZT}=$ flower bud; $\mathrm{ZC}=$ shoot apical meristem.

\section{Identification of differentially expressed cDNA clones}

The recovered TDFs were reamplified with the original primer sets used for cDNAAFLP analysis and examined on $1.5 \%$ agarose gels. The expected sizes ranged from 151 to $695 \mathrm{bp}$. After the isolated TDFs were cloned, five colonies were selected and sequenced for each TDF, generating 42 distinct nucleotide sequences. The sequences were then subjected to homology searches using BLAST against the databases, whereby 26 sequences with lower evalue were obtained (Table 2). In addition, some sequences did not result in any hits, possibly as a result of poor knowledge of the $A$. sinensis genome. 
Table 2. Nucleotide homology of the transcript-derived fragments with known gene sequences in the database using BLASTN algorithm along with their expression patterns.

\begin{tabular}{|c|c|c|c|c|c|}
\hline AFLP fragment & dbEST_Id & GenBank_Accession No. & Homology & E-value & Length \\
\hline A203-1 & 72420447 & HS513742 & Trans-caffeoyl-CoA 3-O-methyltransferase & $8 \mathrm{e}-11$ & 537 \\
\hline A204 & 72420448 & HS513743 & Actin-related protein ARP4 & 0.0035 & 403 \\
\hline A208-1 & 72420450 & HS513745 & Retrotransposon V14 & $1 \mathrm{e}-31$ & 696 \\
\hline $\mathrm{A} 210$ & 72420452 & HS513747 & 1-Deoxy-D-xylulose 5-phosphate reductoisomerase & $7 \mathrm{e}-04$ & 625 \\
\hline A211-1 & 72420453 & HS513748 & $\begin{array}{l}\text { Transposable element gene; pseudogene, } \\
\text { hypothetical protein }\end{array}$ & $\underline{0.89}$ & 502 \\
\hline A214-1 & 72420455 & HS513750 & Isoamylase & $1 \mathrm{e}-40$ & 695 \\
\hline A303 & 72420457 & HS513752 & Transposable element gene & $\underline{7.8 \mathrm{e}-13}$ & 359 \\
\hline A308-1 & 72420458 & HS513753 & Transposable element gene & $4.3 \mathrm{e}-05$ & 240 \\
\hline A3 310 & 72420459 & HS513754 & 15-Cis-zeta-carotene isomerase & $\overline{0.00065}$ & 422 \\
\hline A316-1 & 72420461 & HS513756 & $\begin{array}{l}\text { Daucus carota subsp sativus clone BAC C073D12 } \\
\text { genomic sequence }\end{array}$ & $5 e-11$ & 262 \\
\hline A327-1 & 72420462 & HS513757 & $\begin{array}{l}\text { With NAD kinase activity, the protein was also } \\
\text { shown to bind calmodulin }\end{array}$ & $\underline{2.9 \mathrm{e}-05}$ & 204 \\
\hline A348-1 & 72420463 & HS513758 & Transposable element gene & $\underline{7 \mathrm{e}-08}$ & 151 \\
\hline A $365-1$ & 72420464 & HS513759 & Transposable element gene & $\overline{2.9 \mathrm{e}-25}$ & 354 \\
\hline A314-1 & 72420466 & HS513761 & Galactose oxidase & $\underline{0.0073}$ & 332 \\
\hline A352-1 & 72420467 & HS513762 & $\begin{array}{l}\text { A chloroplast trans-acting factor of the psbD } \\
\text { light-responsive promoter }\end{array}$ & $\underline{0.41}$ & 288 \\
\hline A364-1 & 72420468 & HS513763 & $\begin{array}{l}\text { Solanum lycopersicum strain Heinz } 1706 \\
\text { chromosome } 1 \text { clone hba-168g4 map } 1\end{array}$ & $1 e-16$ & 384 \\
\hline A362-1 & 72420469 & HS513764 & Transposable element gene & $2.7 \mathrm{e}-24$ & 413 \\
\hline A349-1 & 72420471 & HS513766 & $\begin{array}{l}\text { Daucus carota subsp sativus clone BAC C061I05 } \\
\text { genomic sequence }\end{array}$ & $8 \mathrm{e}-18$ & 335 \\
\hline A311-1 & 72420472 & HS513767 & Transposable element gene & $\underline{3.2 \mathrm{e}-29}$ & 384 \\
\hline A353-1 & 72420473 & HS513768 & $\begin{array}{l}\text { Daucus carota subsp sativus clone BAC C } 155 \mathrm{P} 02 \\
\text { genomic sequence }\end{array}$ & $1 \mathrm{e}-23$ & 236 \\
\hline A309-1 & 72420474 & HS513769 & Transposable element gene & $\underline{4.5 \mathrm{e}-05}$ & 442 \\
\hline A325-1 & 72420475 & HS513770 & Transposable element gene & $\underline{\underline{3.1 \mathrm{e}-15}}$ & 330 \\
\hline A351-1 & 72420476 & HS513771 & $\begin{array}{l}\text { Calmodulin-binding protein }(\mathrm{CAM}) \text {, } \\
\text { reverse transcriptase }\end{array}$ & $2 \mathrm{e}-13$ & 310 \\
\hline A241-1 & 72420479 & HS513774 & Transposable element gene & $\underline{0.12}$ & 295 \\
\hline A242-1 & 72420480 & HS513775 & RNA-directed DNA polymerase gene & $5 e-06$ & 269 \\
\hline $238-1$ & 72420481 & HS513776 & P. trichocarpa multidrug/pheromone exporter & $3 e-31$ & 343 \\
\hline
\end{tabular}

\section{Confirmation of differentially expressed fragments using sqRT-PCR}

Among the 26 sequences with lower e-values, 10 sequences were found with clear functions and a close relationship to bolting. Their expressions were analyzed using sqRTPCR with the primer pairs listed in Table 3 . The results showed that they were expressed at a higher level in early bolting $A$. sinensis (Figure 2).

Table 3. Sequences of the primers used for semi-quantitative reverse transcription polymerase chain reaction.

\begin{tabular}{lll}
\hline TDF clone & Forward primer $\left(5^{\prime}-3^{\prime}\right)$ & Reverse primer $\left(5^{\prime}-3^{\prime}\right)$ \\
\hline A203-1 & GCTCGAAGTCCGAATGAATC & ACTGCCCATGCGAAGATAAC \\
A210 & ACGCCTTGATCTTTGCAAGT & TGTAACTGGTTTCCCCCTTG \\
A214-1 & AAATCCAGCCAACCTCCTCT & CCAATCAAGCTGCACTGTGT \\
A310 & GCACTTTTCAAATTGGGTCAA & GCGTGATTGGTATGTGCATC \\
A314-1 & GTTGGCTGTTTGGAAGCTG & ACAACCAACATGTGCACGAC \\
A361-1 & CCCCTCCCCCTCTGTATAGT & TGGATTTGTGGCTTTGATGA \\
A327-1 & TTTTGGCGTGATATGTTTTGT \\
A351-1 & CGGATACGACGTTTTCTGAAG & TGGCAAAAATGTCATGAACC \\
A242-1 & TCCTTGCAAAACGAGCTTCT & ATTTTGATTCCACGCAGCTT \\
A238-1 & TGGCATCTGTGGGAATTACA & TATTCAACCACCCCTTCTCC \\
\hline
\end{tabular}




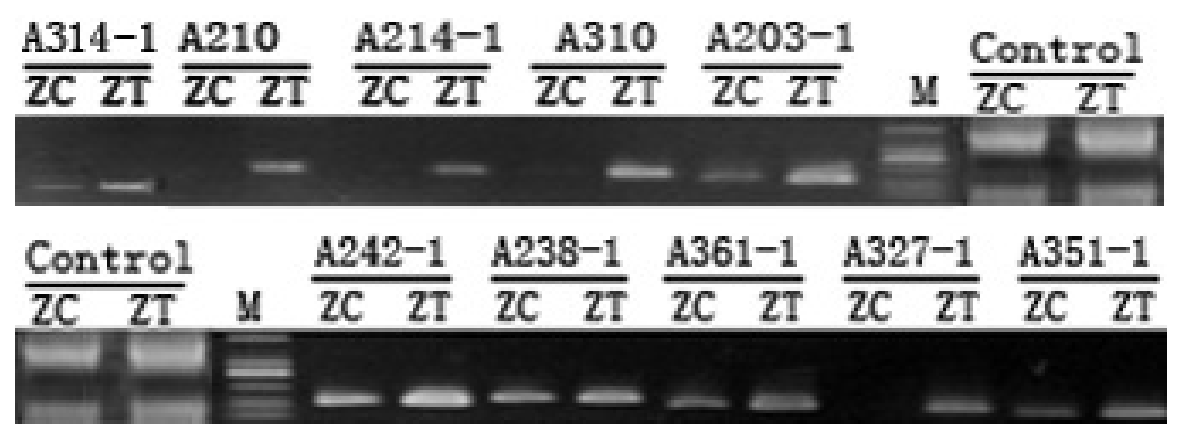

Figure 2. Confirmation by semi-quantitative RT-PCR of the higher level expression of TDFs in the flower bud of early-bolting $A$. sinensis (Oliv.) Diels. ZT $=$ flower bud; $\mathrm{ZC}=$ shoot apical meristem.

\section{DISCUSSION}

cDNA-AFLP, a variation of AFLP derived from RNA fingerprint identification technology, has already become a sophisticated research tool for identifying gene expression differences. In this study, cDNA-AFLP was used to compare the gene expression profiles of flower buds and shoot apical meristems. Twenty-six sequences were obtained, and some might be involved in the control of early bolting. We focused on sequences that were identified to be trans-caffeoyl-coenzyme A (CoA) 3-O-methyltransferase, 1-deoxy-D-xylulose 5-phosphate reductoisomerase, 15-cis-zeta-carotene isomerase, isoamylase, nicotinamide adenine dinucleotide (NAD) kinase, galactose oxidase, calmodulin-binding protein $(\mathrm{CaM})$, and transposable element gene. In all cases, these genes were involved in cellular pathways leading to bolting.

TDF A210, shown in Table 2, is homologous to 1-deoxy-D-xylulose 5-phosphate reductoisomerase, which is related to terpenoid backbone biosynthesis. 1-Deoxy-D-xylulose 5-phosphate reductoisomerase catalyzes the biosynthesis of isoprene, zeatin, monoterpenoid, diterpenoid, carotenoid, porphyrin, chlorophyll, N-glycan, steroids, sesquiterpenoid ubiquinone, and other terpenoid quinones. The hormone zeatin affects plant bolting; zeatin is markedly more effective than kinetin and 6-(3-methylbut-2-enyl) aminopurine in promoting frond expansion and increasing frond number of Spirodela oligorrhiza cultures grown under continuous illumination (Letham, 1967). The hormone gibberellin is a derivative of diterpenoid that plays a central role in modulating diverse processes throughout plant development. Gibberellins are hormones that control growth and a wide variety of plant developmental processes. The gibberellin pathway is one of the four key pathways that affect flowering (Sun and Gubler, 2004).

A242-1 is homologous to calmodulin-binding protein, which is related to CaM. CaM is a ubiquitous multifunctional calcium sensor in all eukaryotes that mediates calcium action by regulating the activity/function of many unrelated proteins. Calcium and CaM are known to play a crucial role in pollen germination and pollen tube growth (Golovkin and Reddy, 2003).

A238-1 is homologous to the ATP binding cassette (ABC) superfamily, which is a large, ubiquitous, and diverse group of proteins, most of which mediate transport across biological membranes. Sequences of more than 20 plant $\mathrm{ABC}$ proteins have been published, and include homologs of P-glycoprotein, multidrug resistance-associated protein and organellar transporters. Recent results indicate that the function of this protein family is not restricted 
to detoxification processes. Plant $\mathrm{ABC}$ transporters have been demonstrated to participate in chlorophyll biosynthesis, formation of $\mathrm{Fe} / \mathrm{S}$ clusters, stomatal movement, and probably ion fluxes; hence, they may play a central role in plant growth and development (Sidler et al., 1998; Møller et al., 2001).

TDF A203-1 is matched with trans-caffeoyl-CoA 3-O-methyltransferase, which is related to metabolic pathways, biosynthesis of secondary metabolites such as flavonoid phenylpropanoid, and phenylalanine metabolism. Homoeriodictyol, capsaicin, syringin, coniferin, guaiacyl lignin, and syringyl lignin are all related to trans-caffeoyl-CoA 3-O-methyltransferase. TDF A310 is homologous to 15-cis-zeta-carotene isomerase, which is related to the metabolic pathways and biosynthesis of secondary metabolites such as carotenoids. TDF A327-1 is homologous to NAD kinase 2, which encodes a protein with NAD kinase activity. A314-1 is homologous to galactose oxidase.

As summarized in Table 2, the early bolting of $A$. sinensis might be related to pollen germination and pollen tube growth, terpenoid backbone biosynthesis, metabolic pathways, biosynthesis of secondary metabolites, and transposable elements. The differentially expressed genes obtained in this study should help to elucidate the molecular basis of early bolting in $A$. sinensis and identify genes that could be targeted to prevent early bolting and increase $A$. sinensis production.

\section{ACKNOWLEDGMENTS}

We are grateful to the National Natural Science Foundation of China (Project \#30801518), the National Science and Technology Pillar Program, Eleventh Five-Year Plan Period (Projects \#2006BA109B05-01 and \#2007BA137B02), and a Project Funded by the Priority Academic Program Development of Jiangsu Higher Education Institutions for support provided for this research.

\section{REFERENCES}

Altschul SF, Madden TL, Schaffer AA, Zhang J, et al. (1997). Gapped BLAST and PSI-BLAST: a new generation of protein database search programs. Nucleic Acids Res. 25: 3389-3402.

Golovkin M and Reddy AS (2003). A calmodulin-binding protein from Arabidopsis has an essential role in pollen germination. Proc. Natl. Acad. Sci. U. S. A. 100: 10558-10563.

Habu Y, Fukada-Tanaka S, Hisatomi Y and Iida S (1997). Amplified restriction fragment length polymorphism-based mRNA fingerprinting using a single restriction enzyme that recognizes a 4-bp sequence. Biochem. Biophys. Res. Commun. 234: 516-521.

Komeda Y (2004). Genetic regulation of time to flower in Arabidopsis thaliana. Annu. Rev. Plant Biol. 55: 521-535.

Letham DS (1967). Regulators of cell division in plant tissues. Planta 74: 228-242.

Lin MH and Qiu YD (2007). Effect of root diameter on early bolting rate and yield in seedling of Angelica sinensis (Oliv.) Diels. Chin. Traditional Herbal Drugs 38: 1386-1389.

Milioni D, Sado PE, Stacey NJ, Roberts K, et al. (2002). Early gene expression associated with the commitment and differentiation of a plant tracheary element is revealed by cDNA-amplified fragment length polymorphism analysis. Plant Cell 14: 2813-2824.

Møller SG, Kunkel T and Chua NH (2001). A plastidic ABC protein involved in intercompartmental communication of light signaling. Genes Dev. 15: 90-103.

Money T, Reader S, Qu LJ, Dunford RP, et al. (1996). AFLP-based mRNA fingerprinting. Nucleic Acids Res. 24: 26162617.

Nanjing University of Chinese Medicine (2006). Dictionary of Chinese Herbal Medicines. 2nd edn. Shanghai Science and Technology Press, 1207-1213. 
Sidler M, Hassa P, Hasan S, Ringli C, et al. (1998). Involvement of an ABC transporter in a developmental pathway regulating hypocotyl cell elongation in the light. Plant Cell 10: 1623-1636.

Sun TP and Gubler F (2004). Molecular mechanism of gibberellin signaling in plants. Annu. Rev. Plant Biol. 55: 197-223. Wang YL, Liang YZ and Chen BM (2007). High-performance liquid chromatography with atmospheric pressure chemical ionization and electrospray ionization mass spectrometry for analysis of Angelica sinensis. Phytochem. Anal. 18: 265-274.

Yang XB, Zhao Y and Lv Y (2008). In vivo macrophage activation and physicochemical property of the different polysaccharide fractions purified from Angelica sinensis (Oliv.) Diels. Carbohydr. Polym. 71: 372-379.

Yanovsky MJ and Kay SA (2003). Living by the calendar: how plants know when to flower. Nat. Rev. Mol. Cell Biol. 4: $265-275$. 